\title{
Detection and Classification of Power Quality Disturbances Based on Hilbert-Huang Transform and Feed Forward Neural Networks
}

\author{
Saeed Alshahrani \\ College of Engineering, Design \\ and Physical Sciences, Brunel \\ University London, UK \\ Saeed.Alshahrani@brunel.ac.uk
}

\author{
Maysam Abbod \\ College of Engineering, Design \\ and Physical Sciences, Brunel \\ University London, UK \\ Maysam.Abbod@brunel.ac.uk
}

\author{
Gareth Taylor \\ College of Engineering, Design and \\ Physical Sciences, Brunel University \\ London, UK \\ Gareth.Taylor@brunel.ac.uk
}

\begin{abstract}
This paper presents a hybrid detection method and classification Technique based on Hilbert-Huang Transform (HHT) and Feed Forward Neural Networks (FFNNs) to improve the efficient delivery and ensure accurate detection of quality disturbances in the electrical power grids. First, quantities characteristics of power quality disturbances (PQDs) are introduced according its parametrical conditions. Thereafter, a detection and recognition algorithm is used for single and multiple disturbances. Then, a decomposition process and features extraction using Empirical Mode Decompensation (EMD) is conducted for each of these distorted waveforms into Intrinsic Mode Functions (IMFs). Finally, these features are constructed using signal amplitude and frequency and then after fed to one of the powerful Artificial Intelligence Techniques in this field for training, evaluating and testing using (FFNNs) classifier to verify and confirm the effectiveness of the detection methodology.
\end{abstract}

Index Terms - Power Quality, Artificial Intelligence, Classification, Hilbert-Huang Transform (HHT), Feed Forward Neural Networks (FFNNs).

\section{INTRODUCTION}

Power quality (PQ) has taken an important role in for electric power utilities and its customers specially power quality disturbances due to the significant increase in nonlinear load. Such problems have become one of the major challenges for engineers and decisions makers where these events are responsible for a large economical loss to power utilities.

Fourier Transform (FT) is the common method used in definitions of power quantities in IEEE standard 14592010[1]. FT is implemented to analysis power quality events by extracting voltage spectrum at fixed frequencies [2] which is adequate to steady state analysis but not able to handle distorted waveforms as in [3]. A derivative of Fourier Transform named Fast Fourier Transform (FFT) is also widely used to power quality analysis where it is considered as time domain version as in [4] but, not suitable to get amplitude and frequency due to leakage as defined in [5]. Another variant of FT is short time Fourier transform (STFT) which divides power signals into small parts where each division can be presumed as stationary signal [6]. However, it is difficult for non-stationary waveforms to be analysed based on STFT as mentioned in [7].

Wavelet Transform (WT) is also a powerful algorithm used in the field of measuring and analysing power quality issues in which it can represent power signals in time and frequency domain and offers decomposition ability to power signals [8]. WT is categorised into three main branches; continuous wavelet transform (CWT) as in [9], stationary wavelet transform (SWT) as in [10] and discrete wavelet transform (DWT). In [11], Authors applied WT to quantify and localize short duration of power quality disturbances. Discrete wavelet transform (DWT) shows its ability to decompose the 10 signals of non-stationary power quality disturbances into 8 different levels of resolution with features extracted in time domain as shown in [12]. It gets more complex where authors in [13] introduced a hybrid method of detection disturbances based on DWT and S-transform. However, it still important to choose a suitable wavelet branch where the computational cost increase with the increase in the filter length.

Hilbert-Huang Transform (HHT) is a recent and advance signal analysis algorithm which is designed for non-stationary signals when time is important. It decomposes the signal into a fixed number of Intrinsic Mode Functions (IMFs). This type of process is called Empirical Mode Decompensation (EMD) which is able provide the user with the amplitudes and frequencies. It is been involved on few cases of power quality issues recently [14]. IMFs are stored from the highest frequency to the lowest one using the EMD decomposing method. Hilbert-Huang Transform has a important role by giving the instantaneous amplitude and frequency for each IMF [15].

Artificial intelligence (AIs) Techniques can be described as the automation of human thinking activities such learning, problem solving, estimation and decision making. AIs Techniques are required in the classification of power quality analysis. It has proven its importance and ability to classify power signal disturbances and handle data extracted from detection and measuring algorithms [16].

Classifiers based on artificial neural networks (ANNs) are used widely in power systems applications [17]. It shows its effectiveness to solve time series data in which it can 
differentiate between features extracted through pattern recognition [18]. An approach of classifying real time data for power quality events is presented in [19]. In [20], Authors proposed quantum neural networks (QNN) as classification system. With three hidden layers, Authors presented a robust ANNs classifier for data extracted of power quality events in smart grids based on DWT algorithm as in [21].

Fuzzy expert system has a significant participation in the field of power quality. Originally, it evaluates information delivered by linguistic values inputs. It has been involved to identify disturbances based on wavelet transform as in [22]. It is also joined to classify these events with FFT as conducted in [23].

Neuro-fuzzy Classification systems have the advantage to resolve conflicts by aggregation where it has self-learning capability among fast computation processes [24]. In terms of power quality analysis, Neuro-fuzzy technique is implemented as classifier for disturbances with their features extracted as in [25].

A support vector machine (SVM) classification system is developed by Vapnik [26]. The theory is based on the supervised statistical learning which can be used effectively for classification purposes. An approach on identifying voltage disturbances is carried out using SVM classifier [27]. SVMs is also has recently a role in the characterization of power quality events as proved in [28].

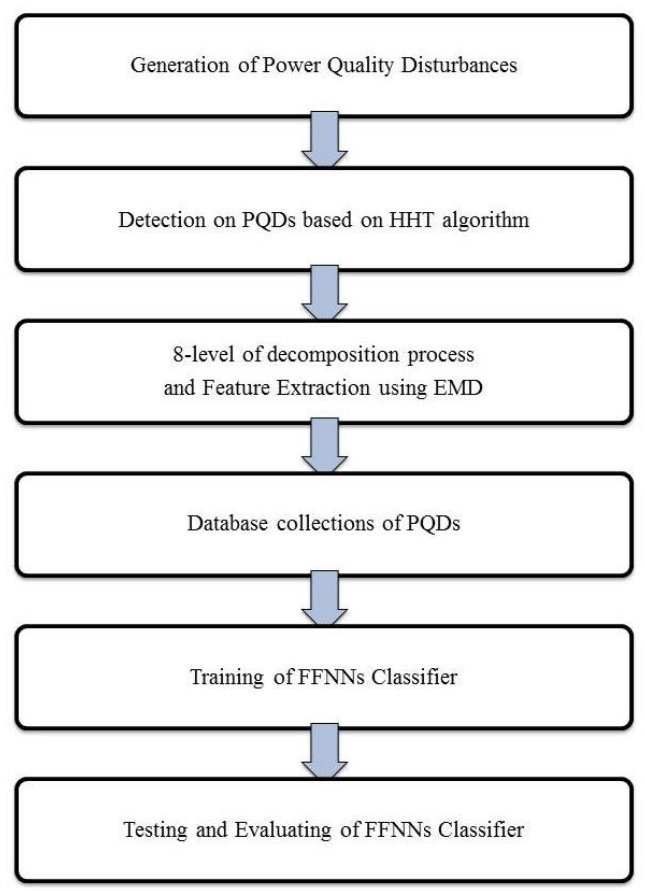

Fig. 1. Flowchart of the detection method and classification Technique

\section{HiLbert-HuANG TrANSFORM}

Hilbert-Huang Transform (HHT) is a recent signal processing algorithm and an effective time frequency method for analysing and detecting the non-stationary signals which contains a powerful feature extraction processer called empirical mode decomposition (EMD).

EMD procedure is able to decompose a non-stationary signal in time domain into an array of sub-signals. Depending on their frequency, these extracted sub-signals are named intrinsic mode functions (IMFs). The highest frequency component of the extracted signal comes as the first IMF where the lowest frequency component comes as the lower one [29]. The sum of IMFs is equal to the original distorted signal as mathematically explained in [30] which can be represented as:

$$
x(t)=\sum_{j=1}^{n} C_{i}+r_{n}
$$

whereas $c j$ represents IMFs from $j=1$ to $n$. then it can be seen that HHT is defining the disturbance signal from real signal to analytical signal as:

$$
y(t)=\int_{-\infty}^{\infty} \frac{x(t)}{t-\tau} d t
$$

where $x(t)$ convolutes with $1 / t$. then, the IMF can be obtained from the HHT analytical signal as:

$$
z(t)=x(t)+i y(t)=a(t) e^{i \theta(t)}
$$

where $a(t)$ represents the amplitude and $\theta(t)$ represents the phase and they are specified as:

$$
\begin{aligned}
& a(t)=\sqrt{x^{2}(t)+y^{2}(t)} \\
& \theta(t)=\arctan (y(t) / x(t))
\end{aligned}
$$

Then after, Frequency of $\omega(t)$ is given by:

$$
\omega(t)=\frac{d \theta(t)}{d t}
$$

As a result, components of time dependent for amplitude and frequency are extracted for every IMF in HHT. But when it comes to Time frequency Domain, then the amplitude can take the formula:

$$
H(\omega, t)=\text { real value } \sum_{i=1}^{n} a(t) e^{i \theta(t)}
$$

Finally, The signal spectrum which delivers the value of the total amplitude participation from each frequency and time domain and resulted as:

$$
h(\omega, t)=\int_{0}^{\mathrm{T}} H(\omega, t) d t
$$

where $\mathrm{T}$ is the signal information length which is provided by the instantaneous amplitude and instantaneous frequency. Each instantaneous amplitude figure is the representative of a specific frequency magnitude with time variation from 0 to $\mathrm{N}-1$ samples. 


\section{Detection Algorithm}

At this stage, it is important to describe power quality disturbances precisely. To achieve that, several standards are developed to characterise these events such as IEEE 1159 standard [31] which describes electromagnetic phenomena, normal operating conditions and variation parameters for voltage, current, power and load supply in electrical power systems. Nevertheless, the European standard EN 50160 specifies thresholds of root mean square (RMS) measurements for voltages and currents when disturbances occur [32]. Finally, ISO standard IEC 61000-4-30 shows methods of reliability in terms of measuring electrical quantities in power systems [33].

In this work, ten power quality disturbances are generated according the standards above especially IEEE 1159 . These disturbances are (sag, swell, harmonics, interruption, flicker, high frequency transient, low frequency transient, sag with harmonics and swell with harmonics). Moreover, the generation stage is implemented successfully for single and multiple events with its parametric limits in Table I. The outputs as shown in Figure 2 of this stage are repeated randomly to contract a database for classification stage.

TABLE I

SignAL MODELLING OF POWER QUALITY DISTURBANCES

\begin{tabular}{|c|c|c|c|}
\hline PQ Disturbances & Class Symbol & Mathematical Models & Parameters \\
\hline Pure Sine & $\mathrm{C} 01$ & $f(t)=A \sin (\omega t)$ & $A=1.0 \quad f=50 \mathrm{~Hz}, \omega=2 \pi 50 \mathrm{rad} / \mathrm{sec}$ \\
\hline Sag & $\mathrm{C} 02$ & $f(t)=A\left(1-\alpha\left(u\left(t-t_{1}\right)-u\left(t-t_{2}\right)\right)\right) \sin (\omega t)$ & $0.1<\alpha<0.9, \quad T \leq t_{2}-t_{1} \leq 9 T$ \\
\hline Swell & $\mathrm{C} 03$ & $f(t)=A\left(1+\alpha\left(u\left(t-t_{1}\right)-u\left(t-t_{2}\right)\right)\right) \sin (\omega t)$ & $0.1<\alpha<0.8, T \leq t_{2}-t_{1} \leq 9 T$ \\
\hline Harmonics & $\mathrm{C} 04$ & $f(t)=A\left(\alpha_{1} \sin (\omega t)+\alpha_{3} \sin (3 \omega t)+\alpha_{5} \sin (5 \omega t)\right.$ & $0.05 \leq \alpha_{3}, \alpha_{5}, \alpha_{7} \leq 0.15, \sum \alpha_{i}^{2}=1$ \\
\hline Interruption & $\mathrm{C} 05$ & $f(t)=A\left(1-\alpha\left(u\left(t-t_{1}\right)-u\left(t-t_{2}\right)\right)\right) \sin (\omega t)$ & $0.9<\alpha<1.0, T \leq t 2-t 1 \leq 9 T$ \\
\hline Flicker & $\mathrm{C} 06$ & $f(t)=A+\alpha_{f} \sin (\omega t)(1+\beta \sin (\gamma \omega t))$ & $0.1 \leq \beta \leq 0.2,0.1 \leq \gamma \leq 0.2$ \\
\hline High frequency transient & $\mathrm{C} 07$ & $f(t)=A\left(\sin (\omega t)+\alpha e^{-t / \lambda} \sin (b \omega t)\right)$ & $\begin{array}{l}20 \leq b \leq 80,0.1 \leq \lambda \leq 0.2 \\
0.1 \leq \alpha \leq 0.9, T \leq t 2-t 1 \leq 3 T \\
1500 \mathrm{~Hz}<f_{n}<2000 \mathrm{~Hz}\end{array}$ \\
\hline Low frequency transient & $\mathrm{C} 08$ & $\left.f(t)=A \sin (\omega t)+\alpha e^{-t / \lambda} \sin (b \omega t)\right)$ & $\begin{array}{l}5 \leq b \leq 20,0.1 \leq \lambda \leq 0.2 \\
0.1 \leq \alpha \leq 0.9, T \leq t 2-t 1 \leq 9 T \\
300 \mathrm{~Hz}<f_{n}<1000 \mathrm{~Hz}\end{array}$ \\
\hline Sag with harmonics & C 09 & $\begin{aligned} f(t)= & A\left(1-\alpha\left(u\left(t-t_{1}\right)-u\left(t-t_{2}\right)\right)\right) \\
& \left.\left(\sin (\omega t)+\alpha_{3} \sin (3 \omega t)+\alpha_{5} \sin (5 \omega t)\right)\right)\end{aligned}$ & $\begin{array}{l}0.1<\alpha<0.9,0.05<\alpha_{3}<0.15,0.05<\alpha_{5}< \\
0.15, T \leq t 2-t 1 \leq 9 T, \sum \alpha_{i}{ }^{2}=1\end{array}$ \\
\hline Swell with harmonics & C 10 & $\begin{aligned} f(t)= & A\left(1+\alpha\left(u\left(t-t_{1}\right)-u\left(t-t_{2}\right)\right)\right) \\
& \left.\left(\sin (\omega t)+\alpha_{3} \sin (3 \omega t)+\alpha_{5} \sin (5 \omega t)\right)\right)\end{aligned}$ & $\begin{array}{l}0.1<\alpha<0.8,0.05<\alpha_{3}<0.15,0.05<\alpha_{5}< \\
0.15, T \leq t 2-t_{1} \leq 9 T, \sum \alpha_{i}{ }^{2}=1\end{array}$ \\
\hline
\end{tabular}

\section{FEATURES EXTRACTION}

In this section, each distorted waveform is decomposed into 8-level of intrinsic mode functions IMFs with the assist of EMD ability. This strategy is based upon the energy of each disturbance generated. The key factors in this stage are the instantaneous amplitude and the instantaneous frequency which obtained and extracted from the original distorted power signal and calculated in groups for targeted disturbances. IMFs of these disturbances are detailed in
Figures 2 to 11 where each disturbance has a unique IMF to be represented as fingerprint which is very important of classification stage taking into account that these disturbances are considered as a Short Duration Variations. 

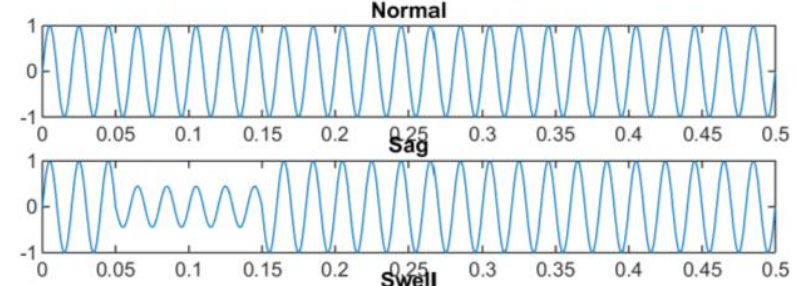

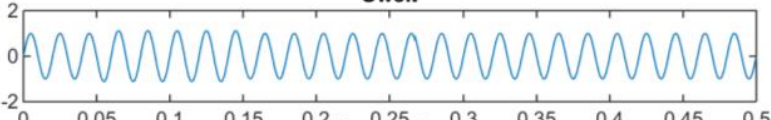
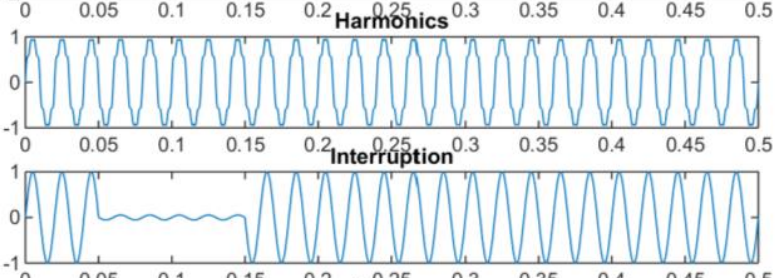

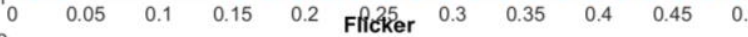

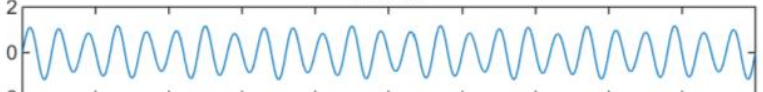

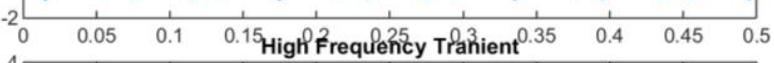

ônAm

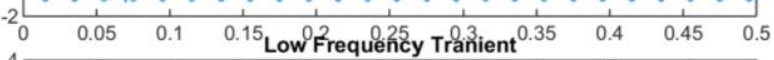

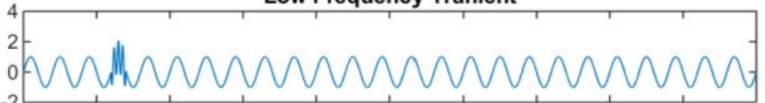

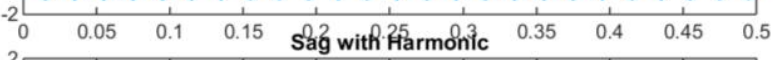

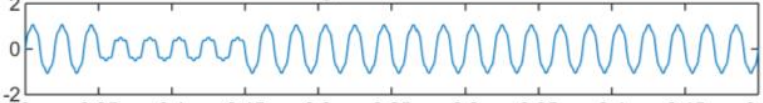

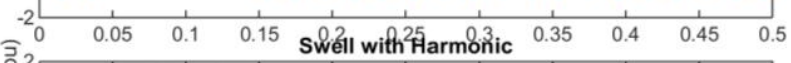

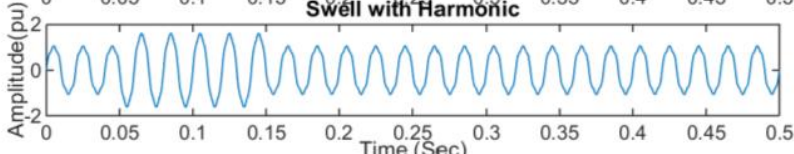

Fig. 3. Waveforms of power quality disturbances
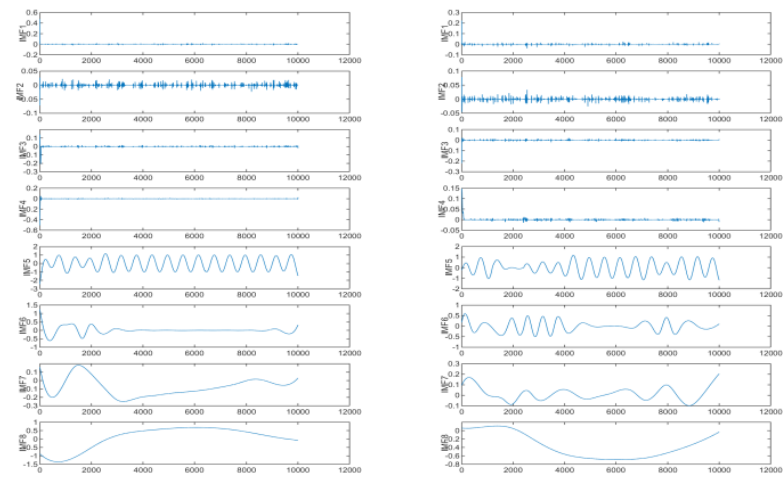

Fig. 4. IMFs for Pure signal

Fig. 5. IMFs for Sag signal

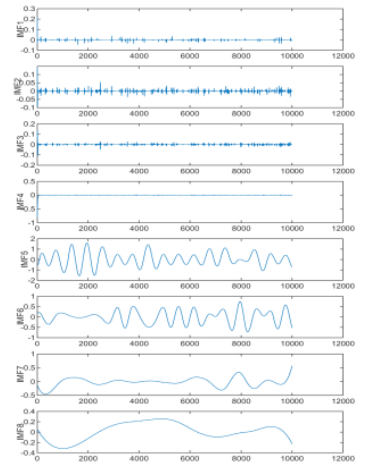

Fig. 6. IMFs for Swell signal

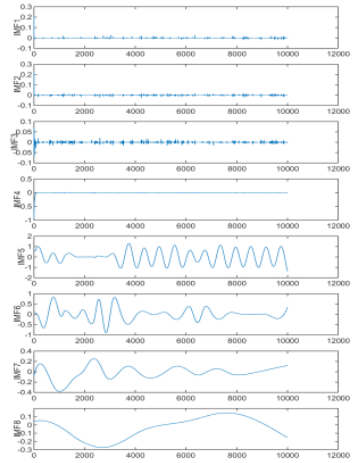

Fig. 6. IMFs for Interruption signal Fig. 7. IMFs for Flicker signal
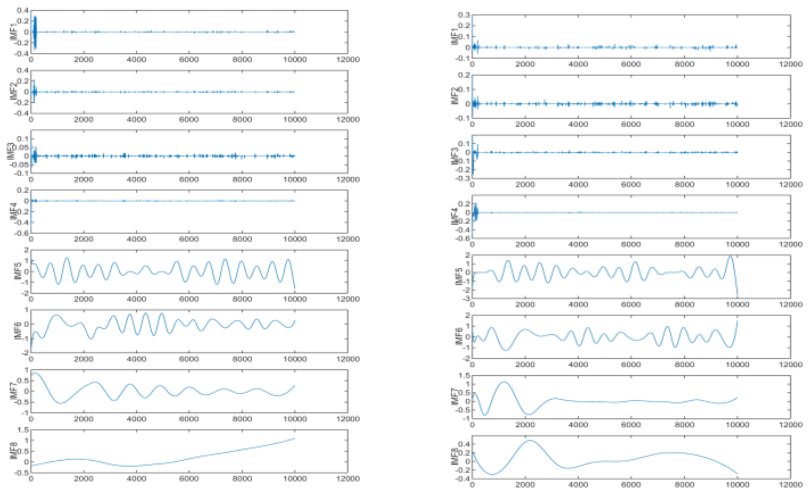

Fig. 8. IMFs High Frequency Transient Fig. 9 IMFs low Frequency Transient

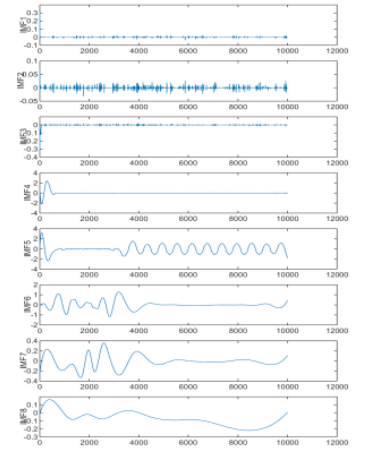

Fig. 10. IMFs Sag with Harmonics
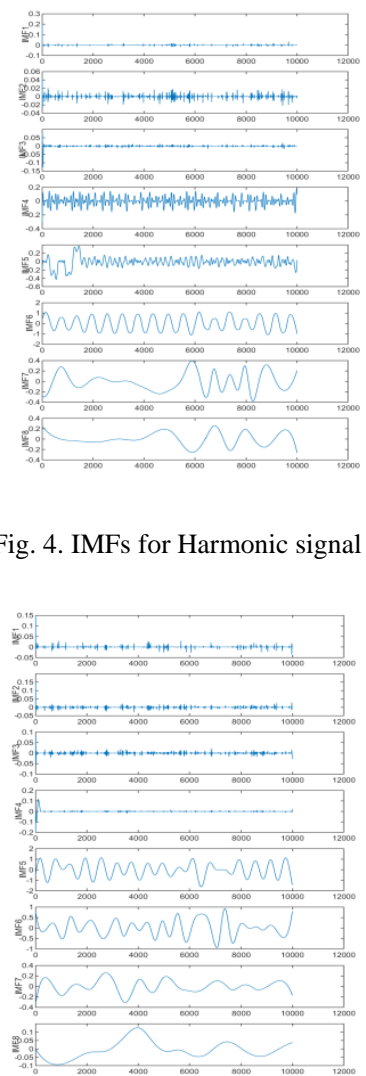

Fig. 4. IMFs for Harmonic signal 


\section{Classification System TechniQue}

In order to evaluate and exam the detection method, a classification system Technique is needed. In this study, Feed Forward Neural Networks (FFNNs) classifier is constructed to promote this stage which has an architecture outlined in Figure 12. A Synthetic database of features extracted for seven PQDs is trained where each one of these events has its own neural.

Network learning is the key factor of Als system. Therefore, the results of these features are fed to FFNNs as inputs and learning procedure is promoted to solve issues attached to database and then solve other learned results. The role of FFNNs is based on multiplying inputs with their weights. Then, results are calculated by mathematical process to find the activation kays of the neural.

In this study, three parts of training are conducted for data collected. First, features of these disturbances are fed to FFNNs according to which pattern recognition as inputs for training. Secondly, data matrix validation part which is done by measuring the network efficiency. Final part is testing the accuracy to ensure the best performance of the neural networks.

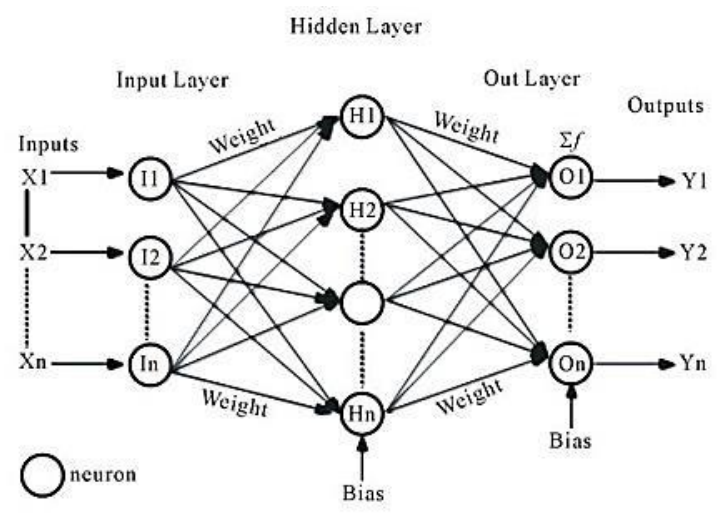

Figure 12. Architecture of FFNNs.

\section{RESUlTS AND DisCUSSION}

Signals of ten cases of most common single and multiple power quality disturbances were generated randomly which covered any possibility of such phenomena in electrical power systems. These signals are then detected successfully by Hilbert-Huang transform (HHT) which is one of the latest and effective methods in the field of signal processing algorithms. A detailed 8-level of decomposition procedure based on EMD is done for each of these events which have information required such as energy components, instantaneous amplitude and instantaneous frequency.

Training of FFNNs for inputs database based on extracted features for distorted signals is built up from 40 random variables. Testing of hidden layers of FFNNs classifier is done by one, two and three hidden layers from 5 to 50 neurons to ensure accuracy and find the best performance of the classifier and better topology in this field. Results of three hidden layers according to the topology [7:15:30:15:7] shows the best accuracy as detailed in Table II as well as the overall accuracy for FFNNs classifier as shown in table II.

TABLE II

ClassifiCATION ACCURACY OF FFNNS With THREE HIDDEN LAYERS

\begin{tabular}{lll}
\hline Neural & Power Quality Disturbances & $\begin{array}{l}\text { Accuracy Rate } \\
(\mathbf{\%})\end{array}$ \\
\hline C 01 & Pure Sine & $99.00 \%$ \\
C 02 & Sag & $96.60 \%$ \\
C 03 & Swell & $98.90 \%$ \\
C 04 & interruption & $98.40 \%$ \\
C 05 & flicker & $100.00 \%$ \\
C 06 & high frequency transient & $97.10 \%$ \\
C 07 & low frequency transient & $97.40 \%$ \\
\hline & Overall Accuracy & $\mathbf{9 8 . 2 0 \%}$ \\
\hline
\end{tabular}

\section{CONCLUSIONS}

This paper presents an approach of a hybrid detection and classification approach based on HHT and FFNNs classifier for power quality disturbances in single and multiple situations. In this work, it has been proved that HHT is able to overcome other transforms limitation especially Fourier transform with non-stationary waveforms which stated in this paper. Thereafter, Features of these disturbances are extracted to 8 levels scenarios of Intrinsic Mode Functions IMFs and based on EMD and a calculation of energy values is done for each disturbance based in its instantaneous amplitude and instantaneous frequency.

FFNNs Classifier is applied for database of a random PQDs signals generated. Results of these data are then analysed, trained and tested to evaluate the accuracy of the detection method and classification Technique. As a result, the overall accuracy of the FFNNs classifier indicates more than $98 \%$ of the performance of HHT and proved robustness of the classifier.

\section{REFERENCES}

[1] IEEE Standard "1459-2010 Power System Instrumentation and Measurements of the IEEE Power \& Energy Society " 2010.

[2] E. Fuchs, B. Trajanoska, S. Orhouzee and H. Renner, "Comparison of wavelet and fourier analysis in power quality," in Electric Power Quality and Supply Reliability Conference (PQ), 2012, 2012, pp. 1-7.

[3] A. Rodriguez, J. Aguado, J. Lopez, F. Martin, F. Munoz and J. Ruiz, "Time-frequency transforms comparison for power quality analysis," in Electricity Market, 2008. EEM 2008. 5th International Conference on European, 2008, pp. 1-6.

[4] F. Zhang, Z. Geng and W. Yuan, "The algorithm of interpolating windowed FFT for harmonic analysis of electric power system," Power Delivery, IEEE Transactions on, vol. 16, pp. 160-164, 2001.

[5] H. Qian, R. Zhao and T. Chen, "Interharmonics analysis based on interpolating windowed FFT algorithm," Power Delivery, IEEE Transactions on, vol. 22, pp. 1064-1069, 2007. 
[6] D. Granados-Lieberman, R. Romero-Troncoso, R. Osornio-Rios, A Garcia-Perez and E. Cabal-Yepez, "Techniques and methodologies for power quality analysis and disturbances classification in power systems: a review," Generation, Transmission \& Distribution, IET, vol. 5, pp. 519-529, 2011

[7] Y. Gu and M. H. Bollen, "Time-frequency and time-scale domain analysis of voltage disturbances," Power Delivery, IEEE Transactions on, vol. 15, pp. 1279-1284, 2000.

[8] S. Santoso, E. J. Powers and W. Grady, "Power quality disturbance data compression using wavelet transform methods," Power Delivery, IEEE Transactions on, vol. 12, pp. 1250-1257, 1997.

[9] L. Angrisani, P. Daponte, M. Apuzzo and A. Testa, "A measurement method based on the wavelet transform for power quality analysis," Power Delivery, IEEE Transactions on, vol. 13, pp. 990-998, 1998.

[10] W. G. Morsi and M. El-Hawary, "A new perspective for the IEEE Standard 1459-2000 via stationary wavelet transform in the presence of nonstationary power quality disturbance," Power Delivery, IEEE Transactions on, vol. 23, pp. 2356-2365, 2008.

[11] C. Xiangxun, "Wavelet-based detection, localization, quantification and classification of short duration power quality disturbances," in Power Engineering Society Winter Meeting, 2002. IEEE, 2002, pp. 931-936.

[12] S. Alshahrani, M. Abbod, B. Alamri and G. Taylor, "Evaluation and classification of power quality disturbances based on discrete wavelet transform and artificial neural networks," in Power Engineering Conference (UPEC), 2015 50th International Universities, 2015, pp. $1-5$.

[13] P. K. Ray, S. R. Mohanty and N. Kishor, "Disturbance detection in grid-connected distributed generation system using wavelet and Stransform," Electr. Power Syst. Res., vol. 81, pp. 805-819, 2011.

[14] M. J. Afroni, D. Sutanto and D. Stirling, "Analysis of nonstationary power-quality waveforms using iterative Hilbert Huang transform and SAX algorithm," Power Delivery, IEEE Transactions on, vol. 28, pp. 2134-2144, 2013

[15] C. Yang, S. Dong, Z. Tong and Y. Wan, "Analysis and research of the transient composite disturbance signal of power system based on HHT," in Intelligent Information Technology Application Workshops, 2008. IITAW'08. International Symposium on, 2008, pp. 1029-1032.

[16] W. R. A. Ibrahim and M. M. Morcos, "Artificial intelligence and advanced mathematical tools for power quality applications: a survey," Power Delivery, IEEE Transactions on, vol. 17, pp. 668-673, 2002.

[17] M. Uyar, S. Yildirim and M. T. Gencoglu, "An effective waveletbased feature extraction method for classification of power quality disturbance signals," Electr. Power Syst. Res., vol. 78, pp. 1747-1755, 2008.

[18] C. Lee and S. Nam, "Efficient feature vector extraction for automatic classification of power quality disturbances," Electron. Lett., vol. 34, pp. 1059-1061, 1998.

[19] I. Monedero, C. Leon, J. Ropero, A. Garcia, J. M. Elena and J. C. Montano, "Classification of electrical disturbances in real time using neural networks," Power Delivery, IEEE Transactions on, vol. 22, pp. 1288-1296, 2007

[20] Z. He, H. Zhang, J. Zhao and Q. Qian, "Classification of power quality disturbances using quantum neural network and DS evidence fusion," European Transactions on Electrical Power, vol. 22, pp. 533-547, 2012.

[21] S. Alshahrani, M. Abbod and B. Alamri, "Detection and classification of power quality events based on wavelet transform and artificial neural networks for smart grids," in 2015 Saudi Arabia Smart Grid (SASG), 2015, pp. 1-6.

[22] M. Chacon, J. Duran and L. Santiesteban, "A wavelet-fuzzy logic based system to detect and identify electric power disturbances," in Computational Intelligence in Image and Signal Processing, 2007. CIISP 2007. IEEE Symposium on, 2007, pp. 52-57.

[23] B. Bizjak and P. Planinšič, "Classification of power disturbances using fuzzy logic," in Power Electronics and Motion Control Conference, 2006. EPE-PEMC 2006. 12th International, 2006, pp. 1356-1360.

[24] K. S. Ajil, P. K. Thapliyal, M. V. Shukla, P. K. Pal, P. C. Joshi and R. R. Navalgund, "A new technique for temperature and humidity profile retrieval from infrared-sounder observations using the adaptive neurofuzzy inference system," Geoscience and Remote Sensing, IEEE Transactions on, vol. 48, pp. 1650-1659, 2010.
[25] V. F. Pires, T. G. Amaral and J. Martins, "Power quality disturbances classification using the 3-D space representation and PCA based neuro-fuzzy approach," Expert Syst. Appl., vol. 38, pp. 11911-11917, 2011.

[26] V. N. Vapnik, "The Nature of Statistical Learning Theory," 1995

[27] G. Lv, X. Wang, H. Zhang and C. Zhang, "PQ disturbances identification based on SVMs classifier," in Neural Networks and Brain, 2005. ICNN\&B'05. International Conference on, 2005, pp. 222226.

[28] B. Biswal, M. Biswal, P. K. Dash and S. Mishra, "Power quality event characterization using support vector machine and optimization using advanced immune algorithm," Neurocomputing, vol. 103, pp. 75-86, 2013.

[29] N. E. Huang, M. C. Wu, S. R. Long, S. S. Shen, W. Qu, P. Gloersen and K. L. Fan, "A confidence limit for the empirical mode decomposition and hilbert spectral analysis," in Proceedings of the Royal Society of London A: Mathematical, Physical and Engineering Sciences, 2003, pp. 2317-2345.

[30] R. Kumar, B. Singh and D. T. Shahani, "Recognition of single-stage and multiple power quality events using Hilbert-Huang transform and probabilistic neural network," Electric Power Components and Systems, vol. 43, pp. 607-619, 2015.

[31] "IEEE Recommended Practice for Monitoring Electric Power Quality," IEEE Std 1159-1995, pp. i, 1995.

[32] B. EN, "50160: 2010 Voltage characteristics of electricity supplied by public electricity networks," British Standards Institution, 2011.

[33] International Electrotechnical Commission, "IEC 61000-4-30: Testing and Measurement Techniques. Power Quality Measurement Methods," International Electrotechnical Commission (IEC): Geneva, Switzerland, 2008. 